\title{
Flight mechanics of a novel guided spin-stabilized projectile concept
}

\author{
F Fresconi $^{1 *}$, G Cooper ${ }^{1}$, I Celmins ${ }^{1}$, J DeSpirito ${ }^{1}$, and M Costello $^{2}$ \\ ${ }^{1}$ U.S. Army Research Laboratory, Aberdeen Proving Ground, Maryland, USA \\ ${ }^{2}$ School of Aerospace Engineering, Georgia Institute of Technology, Atlanta, Georgia, USA
}

The manuscript was received on 28 December 2010 and was accepted after revision for publication on 5 April 2011.

DOI: $10.1177 / 0954410011408385$

\begin{abstract}
Precision-guided munitions are of interest to the Army as a means of both reducing collateral damage and increasing the chance of desired effect with the first round fired. Many technical barriers must be overcome to effectively guide a gun-launched projectile. Gun tubes are rifled to impart the appropriate spin to gyroscopically stabilize a statically unstable projectile. Extremely high spin rates complicate the guidance problem for precision-guided munitions. Manoeuvres achieved through some control mechanism must be actuated at the projectile spin rate. Few control mechanisms have been developed for spin-stabilized projectiles. A novel manoeuvre concept is introduced in this effort. The effectiveness of this concept was investigated through a fundamental derivation of flight mechanics and aerodynamic modelling. This derivation and simulation implementation was verified with existing six degree-of-freedom methods. The manoeuvrability of the airframe and power requirements was assessed by the development of a flight control law. Results suggest sufficient manoeuvrability since the control authority is larger than the ballistic dispersion. The guided airframe exhibited no dynamic flight instabilities. Estimates of the power requirements were within current battery technology and size constraints.
\end{abstract}

Keywords: precision munition, flight mechanics, flight control law, flight stability

\section{INTRODUCTION}

Inducing a manoeuvre in an airframe often relies on producing some type of configrational asymmetry in the flight vehicle. Often, a fin or canard is deflected to create an aerodynamic asymmetry. Obtaining an aerodynamic asymmetry in a gun-launched spin-stabilized projectile is orders-of-magnitude more difficult than in a statically stable airframe. Full-bore projectiles fired from guns often feature an aerodynamic centre-of-pressure which is nose-ward of the centre of gravity. As a result, guns are rifled to impart spin to the projectile to gyroscopically stabilize a statically unstable airframe. The technical difficulty with producing an aerodynamic asymmetry in a spinstabilized projectile is the extremely high actuation

* Corresponding author: U.S. Army Research Laboratory, Aberdeen Proving Ground, MD 21015, USA.

email:frank.fresconi@arl.army.mil rate necessary and also the complex flight dynamics induced by manoeuvring a spin-stabilized projectile. This effort introduces a novel manoeuvre system for spin-stabilized projectiles. Flight mechanics and flight control laws are derived for this concept to assess the flight stability and control authority feasibility for nominal trajectories of a typical $155 \mathrm{~mm}$ artillery projectile.

Control surfaces are often actuated with linear or rotational motors with time constants sufficiently faster than the natural yaw rate or roll rate of a statically stable airframe. Statically unstable airframes have orders-of-magnitude faster yaw and spin rates than fin-stabilized airframes, seriously over-stressing the technologies that have been developed to actuate conventional missiles. A few alternative technologies have been proposed to steer spin-stabilized projectiles; however, most of these means provide modest manoeuvrability. 
Control of spin-stabilized munitions has been explored through implementing pulsed jets. Cooper and Costello [1] derived a linear theory of motion for a spinning projectile with impulsive loads. Jets have also been used on fin-stabilized munitions with lower roll rates to affect course correction [2]. The aerodynamic effect of thrusters (jet interaction) is difficult to determine. The guidance problem for thrusters is also complicated due to the associated flight dynamics and possessing only a finite number of discrete thrusters to remove miss distance.

Flow control has been utilized in manoeuvring spin- and fin-stabilized projectiles. McMichael et al. [3] investigated the Coanda effect on the boattail of a spin-stabilized projectile achieved through extremely high-frequency oscillations of a piezo-electric device. Massey and Silton [4] numerically and experimentally showed that the interaction between fins and pins or flaps at supersonic Mach numbers can produce noticeable trajectory deflections. Wind tunnel and modelling efforts of a fin-stabilized projectile with tail-spoiler microactuators illustrated manoeuvre over a wide range of Mach number [5].

Another technique to manoeuvre a spin-stabilized projectile is to de-spin a part of the body. Costello and Peterson [6] derived a non-linear and linear dynamic model of a dual-rotating body. The flight stability of this concept was determined through metrics such as the gyroscopic stability factor for different inertia weighted spin rates. Special care must be taken in the emplacement of the control mechanism for this concept; however, since little control authority may result with control near the nose, as shown in the non-linear flight analysis of Fresconi and Plostins [7].

Internal moving parts have been proposed to control the flight of spin-stabilized projectiles; however, practical limitations usually preclude trajectory corrections significant enough to fully remove ballistic error sources [8-10]. Past work [11-14] has addressed the flight dynamics and stability of projectiles with internal moving parts. These efforts have focused on developing a theoretical framework and applying that to specific examples.

The complex motion of the concept explored herein must be analysed to assess the control authority and ensure that no strong dynamic instabilities result. To achieve this goal, flight mechanics are derived for this unique situation from first principles. These equations are presented along with an aerodynamic model for the projectile and control mechanism. A flight control law is developed in order to guide the airframe and estimate power requirements. Simulations are performed to determine the feasibility of this concept for further development and testing. The concept in this study and flight mechanics has not been addressed in any past work. This effort is also unique in the theoretical modelling of the effects of both internal (mass) and external (aerodynamic) asymmetries.

This article is organized as follows: flight mechanics derivation and aerodynamic modelling, control algorithm development, followed by simulation results, then conclusions.

\section{MANOEUVRE CONCEPT}

The current concept, shown in Fig. 1 relies on a novel application of conventional, affordable technology. An isometric and side view of a typical spin-stabilized projectile with a deployed flow effector for manoeuvre is shown to the right of Fig. 1. A zoomed-in illustration of the control mechanism is presented in the left side of Fig. 1. Here, one can see a rotary motor which is coupled through a flywheel and clutch to a

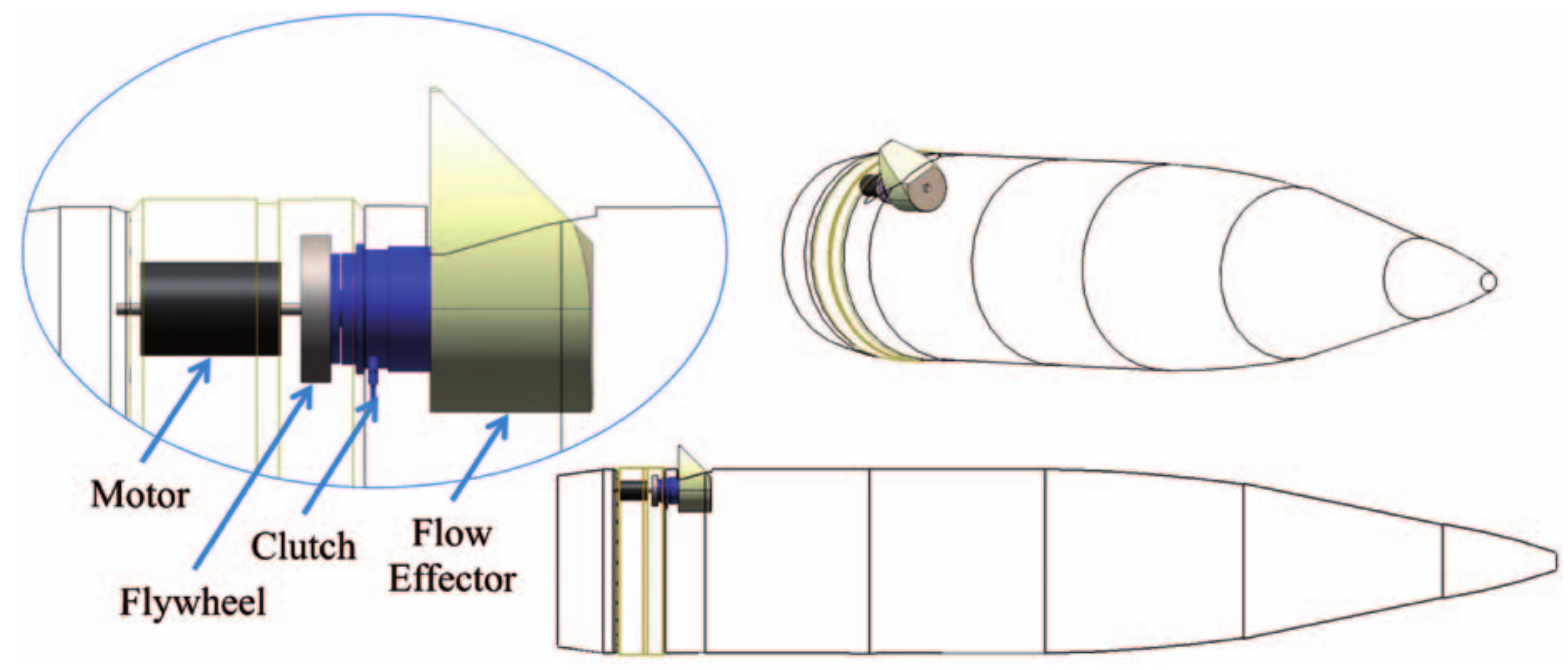

Fig. 1 Novel concept for guided spin-stabilized projectile 
wedge-shaped paddle which extends beyond the surface of the spin-stabilized projectile to create an aerodynamic asymmetry. The motor spins opposite the projectile with an equal magnitude in spin rate. The motor can spin independently when the clutch is disengaged or directly drive the paddle when the flywheel face and clutch are mated. The resulting motion of the paddle when the clutch is engaged is to rotate in and out of the artillery shell in sync with the spin of the projectile, but in the opposite direction. This produces a consistent aerodynamic force and moment which causes a deflection in the trajectory of the projectile in a prescribed roll orientation.

A time sequence of the paddle with respect to the projectile body viewed from the projectile nose in the inertial frame is shown in Fig. 2. Initially (left-most image in sequence), the paddle is stowed within the body. A quarter of the projectile roll cycle later (second image from left in sequence) the motor rotates the flow effector $90^{\circ}$ opposite the roll direction of the projectile such that the paddle is fully deployed. The paddle becomes stowed as the projectile rolls another quarter of a cycle (third image from left in sequence) and the motor continues to roll the paddle another $90^{\circ}$ opposite the direction of the projectile roll. The final phase of the roll cycle (right-most image in sequence) shows the projectile body and paddle rolling another $90^{\circ}$ in opposite directions, respectively, and the paddle remaining stowed. As this cycle repeats, the flow effector maintains a consistent orientation in the inertial frame to produce a manoeuvre. This control mechanism can be used with an appropriate guidance, navigation, and control (GNC) algorithm to achieve a course-correction to minimize miss distance to the target.

This concept is unique in the manner in which the high rate of aerodynamic asymmetry is obtained. Affordable, commercial-off-the-shelf technology for rotary motors provides the necessary frequency response because linear motors cannot meet the requirements. This system can be packaged small enough to fit within a projectile and withstand the extremely high-acceleration loads at launch. The power required to drive this mechanism may be low since no potentially problematic aerodynamic hinge moments, as seen in traditional fin and canard actuation systems, result. The size required for batteries is addressed in this study.

\section{FLIGHT MECHANICS}

The geometry for this problem and associated reference frames are provided in Fig. 3. An inertial reference frame is given by a right-handed coordinate system attached to the earth with origin $\boldsymbol{O}$ and axes denoted by $\boldsymbol{I}_{\boldsymbol{I}}, \boldsymbol{J}_{\boldsymbol{I}}$, and $\boldsymbol{K}_{\boldsymbol{I}}$. Two bodies are described;
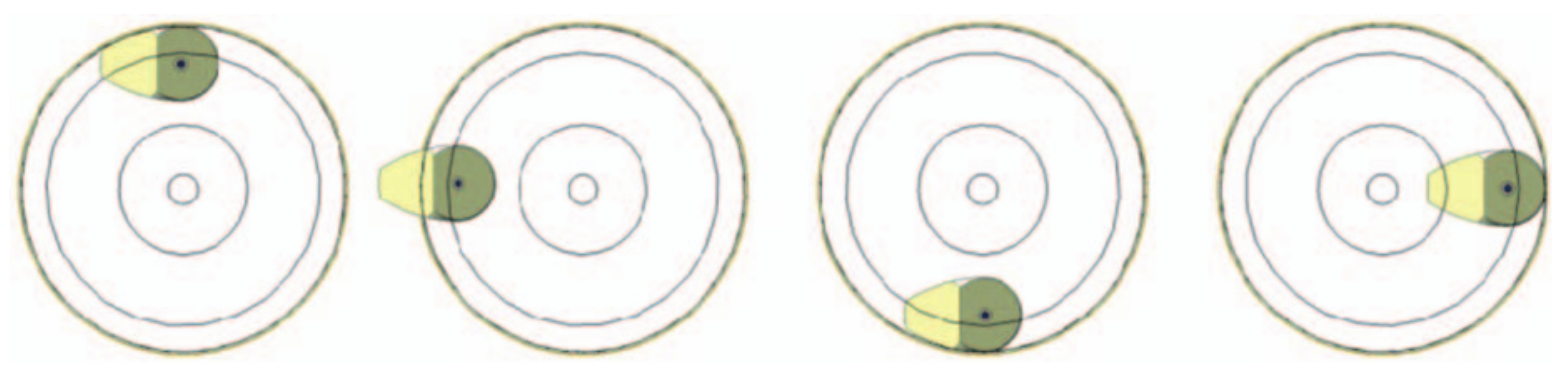

Fig. 2 Snapshots of paddle with respect to projectile throughout roll cycle (viewed in inertial frame from projectile nose)

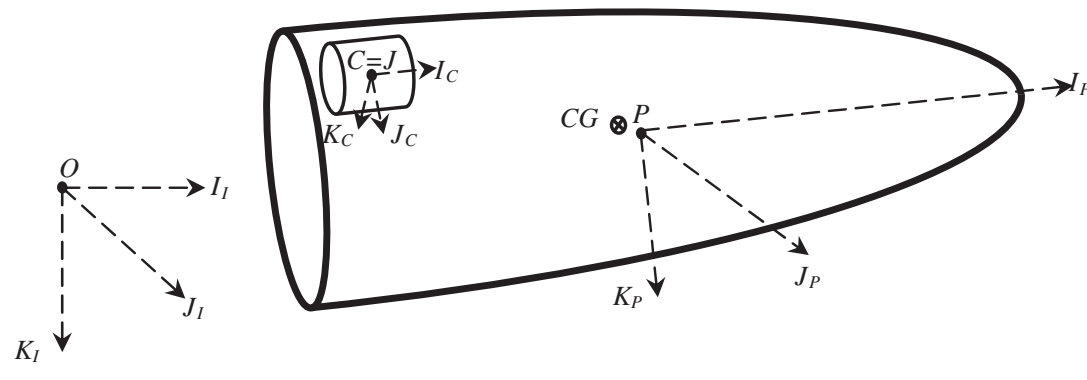

(a)

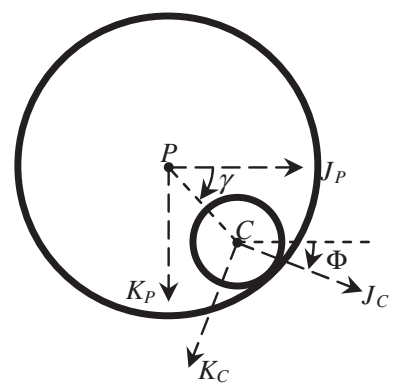

(b)

Fig. 3 Geometry of projectile and control mechanism for multi-body problem: (a) isometric view and (b) viewed from behind 
the projectile and control mechanism. The projectile is fixed with a set of axes $\left(\boldsymbol{I}_{\boldsymbol{P}}, \boldsymbol{J}_{\boldsymbol{P}}\right.$, and $\left.\boldsymbol{K}_{\boldsymbol{P}}\right)$ with origin at the projectile centre of gravity $\boldsymbol{P}$ which is at an arbitrary position and orientation with respect to the inertial frame. $\boldsymbol{I}_{\boldsymbol{P}}$ is selected to be the spin axis of the projectile through point $\boldsymbol{P}$. The control mechanism is modelled as a cylinder which is constrained within the projectile body. Another set of axes $\left(\boldsymbol{I}_{\boldsymbol{C}}, \boldsymbol{J}_{\boldsymbol{C}}\right.$, and $\boldsymbol{K}_{\boldsymbol{C}}$ ) is fixed to the control mechanism body at the centre of gravity $\boldsymbol{C}$. The only degree-of-freedom which the control mechanism has with respect to the projectile is a rotation about the $\boldsymbol{I}_{\boldsymbol{C}}$ axis. The control mechanism has an arbitrary location within the projectile. The projectile and control mechanism bodies are joined at point $\boldsymbol{J}$ which coincides with $\boldsymbol{C}$. An angle $\gamma$ from the $\boldsymbol{J}_{\boldsymbol{P}}$ axis describes the roll orientation of the control mechanism in the projectile body (as seen in Fig. 3(b)). A composite centre of gravity for the multi-body is at point CG.

The standard Euler sequence of rotations $(Z-Y-X)$ is employed to define the orientation of the projectile with respect to the inertial frame in terms of roll $(\phi)$, pitch $(\theta)$, and yaw $(\psi)$ angles. The transformation from inertial to projectile axes is

$$
\begin{aligned}
& {\left[\begin{array}{c}
I_{P} \\
J_{P} \\
K_{P}
\end{array}\right]=\left[\begin{array}{ccc}
c_{\theta} c_{\psi} & c_{\theta} s_{\psi} & -s_{\theta} \\
s_{\phi} s_{\theta} c_{\psi}-c_{\phi} s_{\psi} & s_{\phi} s_{\theta} s_{\psi}+c_{\phi} c_{\psi} & s_{\phi} c_{\theta} \\
c_{\phi} s_{\theta} c_{\psi}+s_{\phi} s_{\psi} & c_{\phi} s_{\theta} s_{\psi}-s_{\phi} c_{\psi} & c_{\phi} c_{\theta}
\end{array}\right]\left[\begin{array}{c}
I_{I} \\
J_{I} \\
K_{I}
\end{array}\right]} \\
& {\left[\begin{array}{c}
I_{P} \\
J_{P} \\
K_{P}
\end{array}\right]=\vec{T}_{I P}\left[\begin{array}{c}
I_{I} \\
J_{I} \\
K_{I}
\end{array}\right]}
\end{aligned}
$$

As seen in Fig. 3(b), an angle $(\Phi)$ represents the roll angle of the control mechanism body. Using this angle, the relationship between projectile and control mechanism frame can be developed.

$$
\left[\begin{array}{c}
I_{C} \\
J_{C} \\
K_{C}
\end{array}\right]=\left[\begin{array}{ccc}
1 & 0 & 0 \\
0 & c_{\Phi} & s_{\Phi} \\
0 & -s_{\Phi} & c_{\Phi}
\end{array}\right]\left[\begin{array}{c}
I_{P} \\
J_{P} \\
K_{P}
\end{array}\right]=\vec{T}_{P C}\left[\begin{array}{c}
I_{P} \\
J_{P} \\
K_{P}
\end{array}\right]
$$

The dynamics for the geometry illustrated in Fig. 3 features seven degrees-of-freedom (DOF): three translational and three rotational for the multi-body plus 1 rotational for the control mechanism. A total of 14 equations must be derived for this seven-DOF problem, seven equations for the kinematics and seven equations for the dynamics. The kinematic equations relate fundamental position and velocity states of motion in the inertial, projectile, and control mechanism reference frames. The dynamic equations provide relationships between the forces and moments and the rate of change of dynamic states of projectile motion. Fourteen states of motion are required since 14 equations of motion are present.
The translational velocity of the multi-body system centre of gravity with respect to an inertial observer $\left(\vec{v}_{C G / I}\right)$ can be written in both the inertial and projectile frames.

$$
\vec{v}_{C G / I}=\dot{x} I_{I}+\dot{y} J_{I}+\dot{z} K_{I}=u I_{P}+v J_{P}+w K_{P}
$$

In this equation, $(\dot{x}, \dot{y}, \dot{z})$ are the three components of the time rate of change of position of the multibody centre of gravity with respect to an observer in the inertial frame written in the inertial frame and $(u, v, w)$ are the three components of the velocity of the multi-body centre of gravity with respect to an observer in the inertial frame written in the projectile frame.

Utilizing this equation along with the transformation matrix above, one can derive the translational kinematics for this problem.

$$
\left[\begin{array}{c}
\dot{x} \\
\dot{y} \\
\dot{z}
\end{array}\right]=\vec{T}_{I P}^{T}\left[\begin{array}{c}
u \\
v \\
w
\end{array}\right]
$$

Additionally, the rotational velocity of the projectile body with respect to an observer in the inertial frame can be written in the inertial and projectile frames

$$
\vec{\omega}_{P / I}=\dot{\phi} I_{P}+\dot{\theta} J_{2}+\dot{\psi} K_{1}=p I_{P}+q J_{P}+r K_{P}
$$

Here, $(\dot{\phi}, \dot{\theta}, \dot{\psi})$ are the time rate of change of the Euler angles and $(p, q, r)$ the three components of the angular velocity of the projectile body with respect to an observer in the inertial frame written in the projectile frame. The $\boldsymbol{J}_{2}$ and $\boldsymbol{K}_{1}$ unit vectors represent a set of interim coordinate systems used for transformations. Performing some coordinate transformations on the above equation enables the rotational kinematic equations to be derived.

$$
\left[\begin{array}{c}
\dot{\phi} \\
\dot{\theta} \\
\dot{\psi}
\end{array}\right]=\left[\begin{array}{ccc}
1 & s_{\phi} t_{\theta} & c_{\phi} t_{\theta} \\
0 & c_{\phi} & -s_{\phi} \\
0 & s_{\phi} / c_{\theta} & c_{\phi} / c_{\theta}
\end{array}\right]\left[\begin{array}{l}
p \\
q \\
r
\end{array}\right]
$$

The final kinematic equation is for the roll angle of the control mechanism and takes the following trivial form due to the straightforward relationship between fundamental position and velocity states of the control mechanism.

$$
\dot{\Phi}=\Omega
$$

Here, $\dot{\Phi}$ is the time rate of change of the control mechanism roll angle with respect to an inertial observer written in the inertial frame and $\Omega$ the rotational rate of the control mechanism.

After developing the kinematic equations, one can collect the 14 states of motion for this problem: $x, y, z, \phi, \theta, \psi, \Phi, u, v, w, p, q, r, \dot{\Phi}$. Some preliminary expressions need to be defined prior to deriving the dynamic equations of motion. The definition of the system mass centre is invoked and the derivative of 
these position vectors with respect to time is taken. Next, the equation for two points on a rigid body is used to arrive at the following relation for the velocity of the projectile centre of gravity with respect to an inertial observer.

$$
\vec{v}_{P / I}=\frac{1}{m}\left[m \vec{v}_{C G / I}-m_{C}\left(\vec{\omega}_{P / I} \times \vec{r}_{P^{\prime} \rightarrow J}\right)\right]
$$

In this equation, $m$ is the mass of the multi-body system, $m_{C}$ the mass of the control mechanism, and $\vec{r}_{P^{\prime} \rightarrow J}$ the position vector from $\mathbf{P}$ to $\mathbf{J}$. The velocity of the projectile centre of gravity is used in the calculations to determine the aerodynamic forces and moments since this is the reference velocity (not the velocity of the multi-body system centre of gravity) used to build aerodynamic coefficients as a function of Mach number and angle-of-attack.

To obtain the translational dynamic model, a free body diagram and kinetic diagram for both the projectile and control mechanism body are drawn and used in Newton's second law. By summing the equations for both bodies and using the relationship between the rate of change of a vector in reference frames in arbitrary relative motion, one can derive the subsequent equation.

$$
\left[\begin{array}{c}
\dot{u} \\
\dot{v} \\
\dot{w}
\end{array}\right]=\left[\begin{array}{ccc}
0 & r & -q \\
-r & 0 & p \\
q & -p & 0
\end{array}\right]\left[\begin{array}{c}
u \\
v \\
w
\end{array}\right]+\left[\begin{array}{c}
X / m \\
Y / m \\
Z / m
\end{array}\right]
$$

Here, $(\dot{u}, \dot{v}, \dot{w})$ are the three components of the acceleration of the multi-body centre of gravity with respect to an observer in the inertial frame written in the projectile frame and $(X, Y, Z)$ the three components of the sum of the external forces.

Using the definition of angular momentum and the relationship between the rate of change of a vector in reference frames in arbitrary relative motion, the rate of change of angular momentum of the projectile body $\left(\frac{{ }^{I} \mathrm{~d} \vec{H}_{P / I}^{P}}{\mathrm{~d} t}\right)$ and control mechanism body $\left(\frac{{ }^{\mathrm{I}} \mathrm{d} \vec{H}_{C / I}^{C}}{\mathrm{~d} t}\right)$ with respect to the inertial frame may be written in the inertial frame.

$$
\begin{aligned}
& \frac{{ }^{I} \mathrm{~d} \vec{H}_{P / I}^{P}}{\mathrm{~d} t}=\frac{{ }^{P} \mathrm{~d} \vec{H}_{P / I}^{P}}{\mathrm{~d} t}+\vec{\omega}_{P / I} \times \vec{H}_{P / I}^{P} \\
& \frac{{ }^{I} \mathrm{~d} \vec{H}_{C / I}^{C}}{\mathrm{~d} t}=\frac{{ }^{P} \mathrm{~d} \vec{H}_{C / I}^{C}}{\mathrm{~d} t}+\vec{\omega}_{P / I} \times \vec{H}_{C / I}^{C}
\end{aligned}
$$

In these expressions, $\frac{{ }^{P} \mathrm{~d} \vec{H}_{P / I}^{P}}{\mathrm{~d} t}$ is the rate of change of angular momentum of the projectile body with respect to the inertial frame written in the projectile frame, $\frac{{ }^{P} \mathrm{~d} \vec{H}_{C / I}^{C}}{\mathrm{~d} t}$ and is the rate of change of angular momentum of the control mechanism body with respect to the inertial frame written in the projectile frame, $\vec{H}_{P / I}^{P}=\vec{I}^{P} \vec{\omega}_{P / I}$ the angular momentum of the projectile body with respect to the inertial frame written in the projectile frame, $\vec{I}^{P}$ the inertial tensor of the projectile body, $\vec{H}_{C / I}^{C}=\vec{T}_{P C}^{T} \vec{I}^{C} \vec{T}_{P C} \vec{\omega}_{C / I}$ the angular momentum of the control mechanism body with respect to the inertial frame written in the projectile frame, $\vec{I}^{C}$ the inertial tensor of the control mechanism body, and $\vec{\omega}_{C / I}=(\dot{\Phi}+p) \boldsymbol{I}_{\boldsymbol{P}}+\boldsymbol{q} \boldsymbol{J}_{\boldsymbol{P}}+\boldsymbol{r} \boldsymbol{K}_{\boldsymbol{P}}$ the rotational velocity of the control mechanism body with respect to the inertial frame written in the projectile frame.

Newtonian kinetics is applied about the joint point $\boldsymbol{J}$ to derive the rotational dynamic equations for the projectile. The rate of change of the multi-body system angular momentum is set equal to the sum of moments about the joint point $\boldsymbol{J}$. The definition of system angular momentum is used to arrive at the following equation.

$$
\begin{aligned}
& \frac{{ }^{I} \mathrm{~d} \vec{H}_{P / I}^{P}}{\mathrm{~d} t}+\vec{r}_{J \rightarrow P} \times m_{P} \vec{a}_{P / I}+\frac{{ }^{I} \mathrm{~d} \vec{H}_{C / I}^{C}}{\mathrm{~d} t}+\vec{r}_{J \rightarrow C} \times m_{C} \vec{a}_{C / I} \\
& \quad=\vec{M}_{P}+\vec{r}_{J \rightarrow P} \times \vec{F}_{P}+\vec{M}_{C}+\vec{r}_{J \rightarrow C} \times \vec{F}_{C}
\end{aligned}
$$

Here, $m_{p}$ is the mass of the projectile, $\vec{a}_{P / I}$ acceleration of the projectile centre of gravity with respect to an observer in the inertial frame written in the projectile frame, $\vec{r}_{J \rightarrow C}$ the position vector from $J$ to $C, \vec{a}_{C / I}$ the acceleration of the control mechanism centre of gravity with respect to an observer in the inertial frame written in the projectile frame, $\vec{M}_{P}$ the three components of the sum of the external moments on the projectile body, $\vec{F}_{P}$ the three components of the sum of the external forces on the projectile body, $\vec{M}_{C}$ the three components of the sum of the external moments on the control mechanism body, and $\vec{F}_{C}$ the three components of the sum of the external forces on the control mechanism body. Using the translational dynamic equation, this equation can be further simplified.

$$
\frac{{ }^{I} \mathrm{~d} \vec{H}_{P / I}^{P}}{\mathrm{~d} t}+\frac{I \mathrm{~d} \vec{H}_{C / I}^{C}}{\mathrm{~d} t}=\vec{M}_{P}+\vec{M}_{C}
$$

A final dynamic equation is needed to close the system. It is necessary to choose the control mechanism rotational dynamic equation in the $\boldsymbol{I}_{\boldsymbol{C}}$-direction since this is the stated degree of freedom of the control mechanism with respect to the projectile. A dot product is taken between $\boldsymbol{I}_{\boldsymbol{C}}$ and the terms in the equation above involving the control mechanism to obtain the final equation.

$$
I_{C} \bullet \frac{{ }^{I} \mathrm{~d} \vec{H}_{C / I}^{C}}{\mathrm{~d} t}=I_{C} \bullet \vec{M}_{C}
$$

In this equation, $I_{C} \bullet \vec{M}_{C}=K_{F} \dot{\Phi}+K_{T} i, K_{F}$ is the friction coefficient of the system, $K_{T}$ the torque constant of the motor, and $i$ the current drawn by the motor. Here, 
one can see that the battery can be sized by integrating the current history over the entire flight.

\section{AERODYNAMIC MODELLING}

The aerodynamic forces and moments, along with the conservative body force (gravity), provide the forcing functions to the dynamic equations of motion. The aerodynamic model was separated into terms involving the projectile and those involving the control mechanism when the flow effector was exposed to the airstream (i.e. during portions of the roll cycle of the control mechanism). Forces acting on the projectile consist of axial force, normal force, and, due to complex flow phenomena for spin-stabilized projectiles, Magnus force.

$$
\vec{F}_{P}=Q S\left[\begin{array}{c}
-C_{X_{0}}+C_{X_{2}} \frac{v_{P}^{2}+w_{P}^{2}}{V} \\
-C_{N_{\alpha}} \frac{v_{P}}{V}+C_{Y_{p \alpha}} \frac{w_{P}}{V} \frac{p d}{2 V} \\
-C_{N_{\alpha}} \frac{w_{P}}{V}-C_{Y_{p \alpha}} \frac{v_{P}}{V} \frac{p d}{2 V}
\end{array}\right]
$$

In this equation, $Q$ is the dynamic pressure, $S$ the aerodynamic reference area, $C_{X_{0}}$ the zero-yaw axial force coefficient, $C_{X_{2}}$ the yaw-squared axial force coefficient, $C_{N_{\alpha}}$ the normal force derivative coefficient, $C_{Y_{p \alpha}}$ the Magnus force coefficient, and $V=\sqrt{u_{P}^{2}+v_{P}^{2}+w_{P}^{2}}$ the total velocity of the projectile.

Pitching moment, pitch damping moment, Magnus moment, and roll damping moment act on the projectile. The pitching moment and Magnus moment are defined in this effort as moment arms crossed with the normal force and Magnus force terms above.

$$
\vec{r}_{P \rightarrow C P}=\frac{C_{m_{\alpha}}}{C_{N_{\alpha}}} d
$$

Here, $\vec{r}_{P \rightarrow C P}$ is the vector from the projectile centre of gravity to the aerodynamic centre of pressure and $C_{m_{\alpha}}$ the pitching moment derivative coefficient.

The Magnus moment coefficient is highly nonlinear with angle-of-attack. For this reason, the expression for the Magnus moment arm is given experimentally as a Taylor series expansion with order according to the Maple-Synge hypothesis for symmetry of projectiles [15].

$$
\vec{r}_{P \rightarrow C M}=\vec{r}_{C M_{0}}+\vec{r}_{C M_{2}} \bar{\alpha}^{2}+\vec{r}_{C M_{4}} \bar{\alpha}^{4}
$$

This expression features the vector from the projectile centre of gravity $\vec{r}_{P \rightarrow C M}$ and total angle-of-attack $\bar{\alpha}=\arcsin \left(\sqrt{v_{P}^{2}+w_{P}^{2}} / V\right)$ along with zeroth-, second-, and fourth-order terms in angle-of-attack $\left(\vec{r}_{C M_{0}}, \vec{r}_{C M_{2}}, \vec{r}_{C M_{4}}\right)$. Note that the terms in the expansion do not have the same units to remain dimensionally consistent. With these relationships for the moment arms, the projectile aerodynamic moments can be shown.

$$
\begin{aligned}
\vec{M}_{P}= & \vec{r}_{P \rightarrow C P} \times Q S\left[\begin{array}{c}
-C_{X_{0}}+C_{X_{2}} \frac{v_{P}^{2}+w_{P}^{2}}{V} \\
-C_{N_{\alpha}} \frac{v_{P}}{V} \\
-C_{N_{\alpha}} \frac{w_{P}}{V}
\end{array}\right] \\
& +\vec{r}_{P \rightarrow C M} \times Q S\left[\begin{array}{c}
0 \\
C_{Y_{p \alpha}} \frac{w_{P}}{V} \frac{p d}{2 V} \\
-C_{Y_{p \alpha}} \frac{v_{P}}{V} \frac{p d}{2 V}
\end{array}\right] \\
& +Q S d\left[\begin{array}{c}
C_{l_{p}} \frac{p d}{2 V} \\
\left(C_{m_{q}}+C_{m_{\dot{\alpha}}}\right) \frac{q d}{2 V} \\
\left(C_{m_{q}}+C_{m_{\dot{\alpha}}}\right) \frac{r d}{2 V}
\end{array}\right]
\end{aligned}
$$

The roll damping coefficient is $C_{l_{p}}$ and the pitch damping coefficient is $\left(C_{m_{q}}+C_{m_{\dot{\alpha}}}\right)$.

Notice that the projectile features symmetric aerodynamics; therefore, the asymmetry necessary to produce a manoeuvre is generated by the control mechanism. All aerodynamic coefficients for the projectile are functions of Mach number. These coefficients were obtained in the Army Research Laboratory's Transonic Experimental Facility spark range at the Aberdeen Proving Ground [16].

The aerodynamics of the control mechanism is of particular interest as this provides the necessary course correction to remove miss distance. The control effector is modelled as an axial force coefficient $\left(C_{X C}\right)$, normal force coefficient $\left(C_{N C}\right)$, and pitching moment coefficient $\left(C_{m C}\right)$ as a function of Mach number and local angle-of-attack in the plane of the control mechanism $\left(\alpha^{\prime}\right)$. To obtain local angle-ofattack for an arbitrarily emplaced control mechanism in roll angle, the following expression is utilized

$$
\alpha^{\prime}=c_{\gamma} \frac{v_{P}}{V}+s_{\gamma} \frac{w_{P}}{V}
$$

Static computational fluid dynamics (CFD) simulations of the flow effector attached to the projectile at various Mach numbers and pitch and yaw angle-ofattack combinations were performed. The full parameter range was not investigated with CFD; however, the data available were fit to a Gaussian distribution in angle-of-attack to complete the database. The reasoning for applying the Gaussian distribution was that as the projectile pitches in the plane of the flow effector the control effectiveness is reduced due to shadowing flow interactions. This modelling approach should provide conservative estimates of control authority. 
A more in-depth characterization and optimization of the flow effector would follow based on the feasibility recommendations from this study.

A sample of the control aerodynamics used for the present effort is given in Figs 4 to 6. These plots show the control axial force coefficient (Fig. 4), control normal force coefficient (Fig. 5), and control pitching moment coefficient (Fig. 6) as a function of angle-ofattack for five different Mach spannings from subsonic to supersonic. The control aerodynamic coefficients are an order-of-magnitude or more lower than the corresponding projectile coefficient.

The control mechanism aerodynamic forces and moments are

$$
\begin{aligned}
& \vec{F}_{C}=\left[\begin{array}{ccc}
1 & 0 & 0 \\
0 & c_{\gamma} & -s_{\gamma} \\
0 & s_{\gamma} & c_{\gamma}
\end{array}\right]\left[\begin{array}{c}
-Q S C_{X C} \\
-Q S C_{N C} \\
0
\end{array}\right] \\
& \vec{M}_{C}=\left[\begin{array}{ccc}
1 & 0 & 0 \\
0 & c_{\gamma} & -s_{\gamma} \\
0 & s_{\gamma} & c_{\gamma}
\end{array}\right]\left[\begin{array}{c}
0 \\
0 \\
Q S d C_{m C}
\end{array}\right]
\end{aligned}
$$

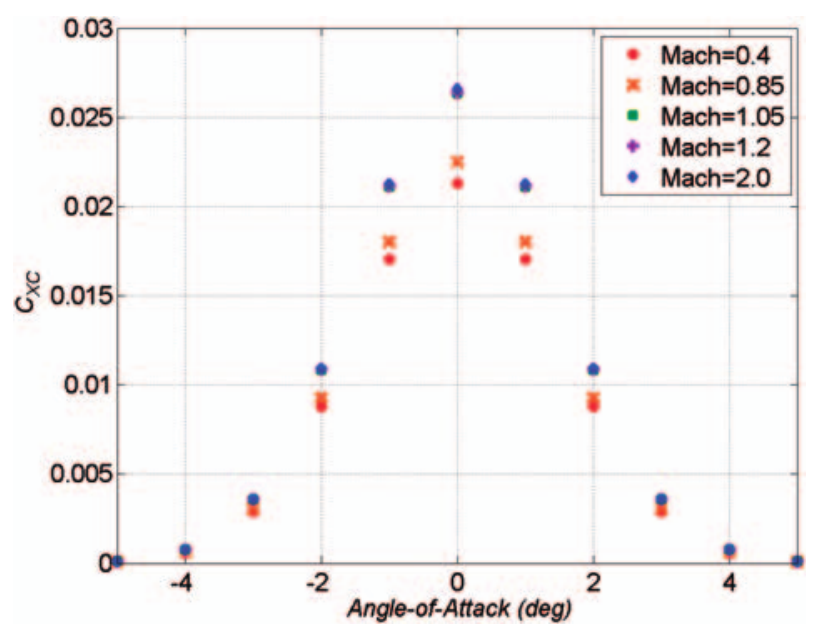

Fig. 4 Control axial force coefficients

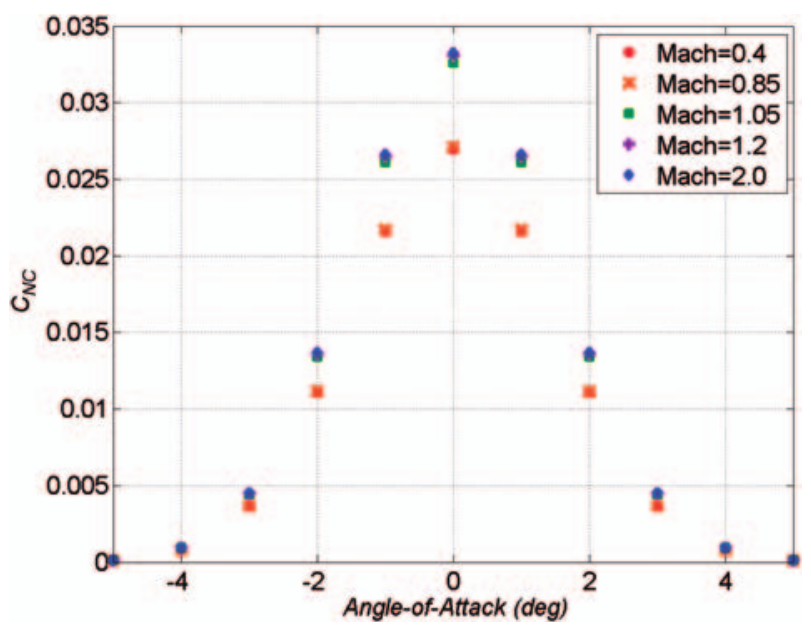

Fig. 5 Control normal force coefficients
Finally, the gravity forces $\left(\vec{F}_{G}\right)$ expressed in the projectile frame are given.

$$
\vec{F}_{G}=\vec{T}_{I P}\left[\begin{array}{c}
0 \\
0 \\
m g
\end{array}\right]
$$

\section{FLIGHT CONTROL ALGORITHM}

The manoeuvre scheme outlined in this effort enables guidance commands to remove miss distance in the form of a roll orientation. The control is essentially discrete since the flow effector is either exposed or stowed; there is no means of continuously varying the magnitude of the control effort. The flight control algorithm developed for this concept tracks roll orientation and deploys the flow effector when desired via engaging the clutch mechanism. The exact

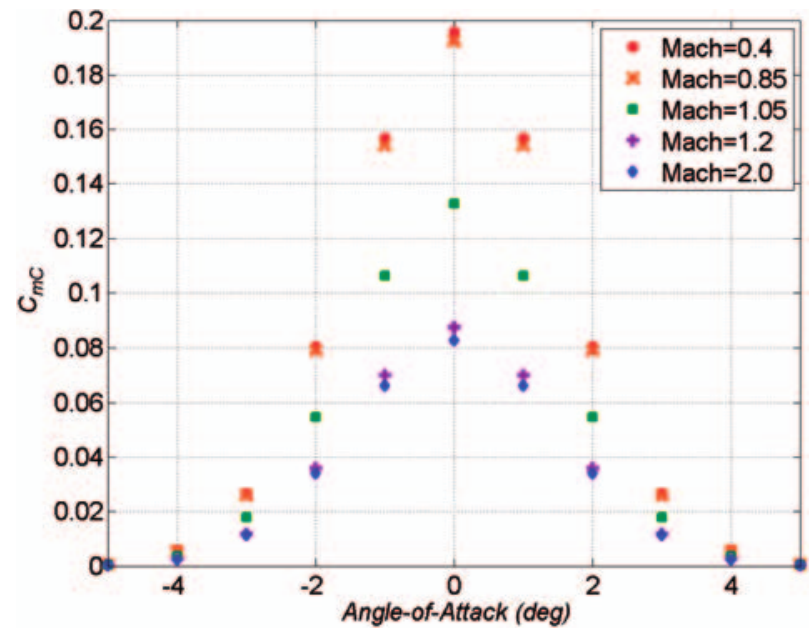

Fig. 6 Control pitching moment coefficients

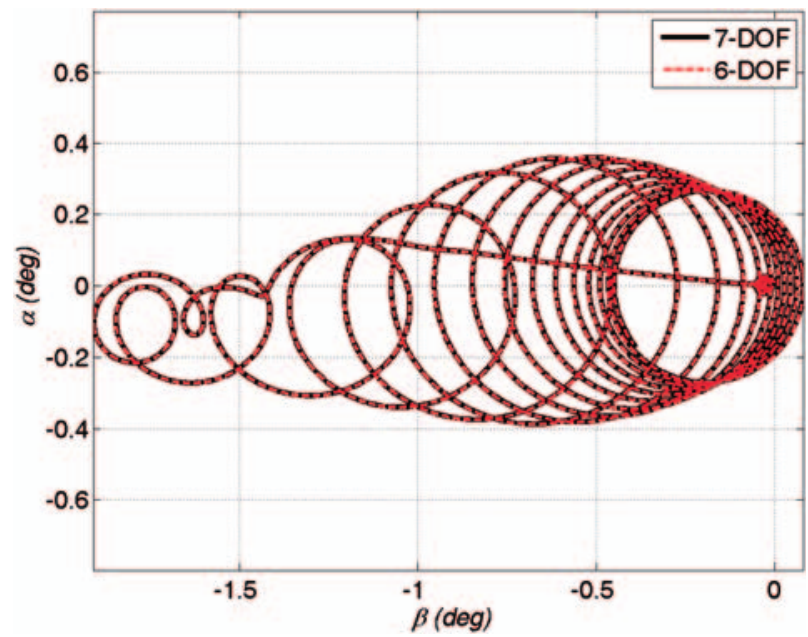

Fig. 7 Verification of angular motion history throughout entire flight 
mechanism by which the flow effector is engaged to the motor is not examined in this effort. Other means, such as spinning the motor up and down when necessary, could be employed. Thus, the flight controller turns the control fully on or off at a prescribed roll orientation $\left(\phi_{C M D}\right)$.

The states necessary to achieve this flight control are projectile roll orientation and control mechanism roll orientation. Projectile roll orientation could be provided by methods such as a global positioning system receiver with an upfinding algorithm or observations of the earth's magnetic field with magnetometers. The control mechanism roll orientation could be provided with relative ease through an optical encoder mounted to the motor. These data are assumed perfect for the purposes of this flight dynamic study.

A simple proportional-derivative controller was found to perform satisfactorily to manoeuvre this projectile. For the derivative terms, the projectile and control mechanism roll orientations are differentiated. This controller issues current commands to the motor to track a given roll orientation. The control law is expressed below.

$$
i=K_{P}\left[-\left(\phi-\phi_{C M D}\right)-\Phi\right]+K_{D}[-\dot{\phi}-\dot{\Phi}]
$$

The proportional gain is $\left(K_{P}\right)$ and the derivative gain is $\left(K_{D}\right)$. It is trivial to see that this control law seeks to rotate the motor in the opposite direction of the projectile spin with a prescribed phase offset to manoeuvre the projectile in the desired direction. The specifications for a direct current brushless motor $\left(K_{T}=\right.$ $0.00823 \mathrm{Nm} / \mathrm{A}$ ) that has been experimentally verified to be gun-hardened was used in the simulations.

\section{RESULTS}

The flight mechanics, aerodynamic modelling, and flight control laws were built into a simulation environment. A complete flight control feedback loop was established by numerically differentiating the nonlinear equations of motion in a state-space form with the current signal needed for the $I_{C}$-direction rotational dynamic equation for the control mechanism coming from the control law shown above. The simulation decides when the flow effector is deployed or stowed based on the roll orientation of the control mechanism body with respect to the projectile body.

The first step in the analysis was to verify the nonlinear flight mechanics for the seven-DOF with a sixDOF model. A ballistic flight was obtained for the seven-DOF by running the simulation with no current input to the motor. Solid modelling of the projectile and control mechanism was undertaken to increase fidelity of the geometry and provide mass and inertial characteristics. Physical properties of a $155 \mathrm{~mm}$ artillery projectile and the control mechanism with and without the flow effector engaged to the driving motor are presented in Table 1. These data were used in simulations performed with a quadrant elevation of $45^{\circ}$ and muzzle velocity of $821 \mathrm{~m} / \mathrm{s}$. Some sample flight dynamics are presented.

Figure 7 shows the angular motion history over the entire flight for the seven-DOF (solid black line) and the six-DOF (dashed red line). The pitch angle-ofattack is $\alpha=\frac{w_{P}}{V}$ and the yaw angle-of-attack is $\beta=\frac{\nu_{P}}{V}$. The projectile was launched with no tip-off. A small pitch angle-of-attack was produced since the spin axis of the projectile lags the trajectory curvature. This phenomenon, along with the fact that the pitching moment is in front of the centre of gravity and the projectile is gyroscopically stabilized, produces the yaw of repose. Repose is evident in the yaw angle-of-attack increasing to almost $-2^{\circ}$ near apogee. When viewed from behind the gun, repose is the yawing of the projectile to the right which generates a normal force in that direction and ultimately drifts the centre of gravity motion to the right. The yaw of repose decreases after apogee.

As the projectile flies through transonic Mach numbers, the non-linear Magnus moment becomes manifest as a weak instability in the form of a coning or limit cycle angular motion that persists through the

Table 1 Physical properties of projectile and control mechanism

\begin{tabular}{llll}
\hline Physical Property & Projectile & $\begin{array}{l}\text { Control mechanism with } \\
\text { flow effector engaged }\end{array}$ & $\begin{array}{l}\text { Control mechanism without } \\
\text { flow effector engaged }\end{array}$ \\
\hline Mass $(\mathrm{kg})$ & 46.1725 & 0.042635 & 0.016603 \\
Diameter $(\mathrm{m})$ & 0.155 & 0.05588 & 0.0254 \\
Length (m) & 0.843 & 0.06106 & 0.03429 \\
Centre of gravity, $\left.I_{\mathrm{P}} \mathrm{m}\right)$ & 0.290 & 0.107 & 0.095 \\
Centre of gravity, $J_{\mathrm{P}}(\mathrm{m})$ & 0.0 & 0.05974 & 0.05969 \\
$I_{X X}\left(\mathrm{~kg}-\mathrm{m}^{2}\right)$ & 0.17061 & $7.0728 \mathrm{e}-6$ & $8.597 \mathrm{e}-7$ \\
$I_{Y Y}\left(\mathrm{~kg}-\mathrm{m}^{2}\right)$ & 2.03385 & $1.0113 \mathrm{e}-5$ & $1.7828 \mathrm{e}-6$ \\
$I_{Z Z}\left(\mathrm{~kg}-\mathrm{m}^{2}\right)$ & 2.03385 & $1.143 \mathrm{e}-5$ & $1.7828 \mathrm{e}-6$ \\
$I_{X Y}=I_{Y X}\left(\mathrm{~kg}-\mathrm{m}^{2}\right)$ & 0.0 & $4.012 \mathrm{e}-7$ & 0.0 \\
$I_{X Z}=I_{Z X}\left(\mathrm{~kg}-\mathrm{m}^{2}\right)$ & 0.0 & 0.0 & 0.0 \\
$I_{Y Z}=I_{Z Y}\left(\mathrm{~kg}-\mathrm{m}^{2}\right)$ & 0.0 & 0.0 & 0.0 \\
\hline
\end{tabular}


subsonic Mach regime with amplitude less than half a degree. Comparing the seven-DOF and six-DOF curves shows an agreeable match; therefore, the derivation of the flight mechanics for this application and the simulation implementation are validated.

The flight stability and the control authority were examined by performing manoeuvres in the four cardinal directions (up, right, down, and left, when viewed from behind the gun) and observing the flight vehicle states. The manoeuvre direction was varied by changing the value of $\phi_{C M D}$ in the control law from $0^{\circ}$ to $360^{\circ}$ in $90^{\circ}$ increments to obtain the four cases. Inspection of Fig. 2 shows that the flow effector is exposed for $180^{\circ}$ of roll orientation; therefore, $180^{\circ}$ was used as the roll window for deployment in the simulations. Future studies will focus on characterizing the aerodynamics of the flow effector for different degrees of exposed surface and shape optimization for necessary control authority; the current effort seeks only to demonstrate the feasibility for this future analysis. For each case, the motor was commanded to track roll orientation from launch and the flow effector rotated in and out of the projectile body at the prescribed roll orientation from $10 \mathrm{~s}$ after launch until impact.

The trajectories in the vertical plane are shown in Fig. 8. The sign of the $\boldsymbol{K}_{\boldsymbol{I}}$ axis was reversed from the coordinate system shown in Fig. 3 to provide the results in Fig. 8 in a more natural manner (i.e. concave down). The ballistic flight reaches almost $8000 \mathrm{~m}$ in altitude and flies over $22000 \mathrm{~m}$ downrange. A range extension is produced by the up case and the down case is a range decrease. Subtracting the downrange distance for the down case from the up case results in almost $700 \mathrm{~m}$ of control authority using the control aerodynamics in Figs 4 to 6 . Thus, a target within this manoeuvre footprint in the downrange direction could be successfully engaged. The left and right

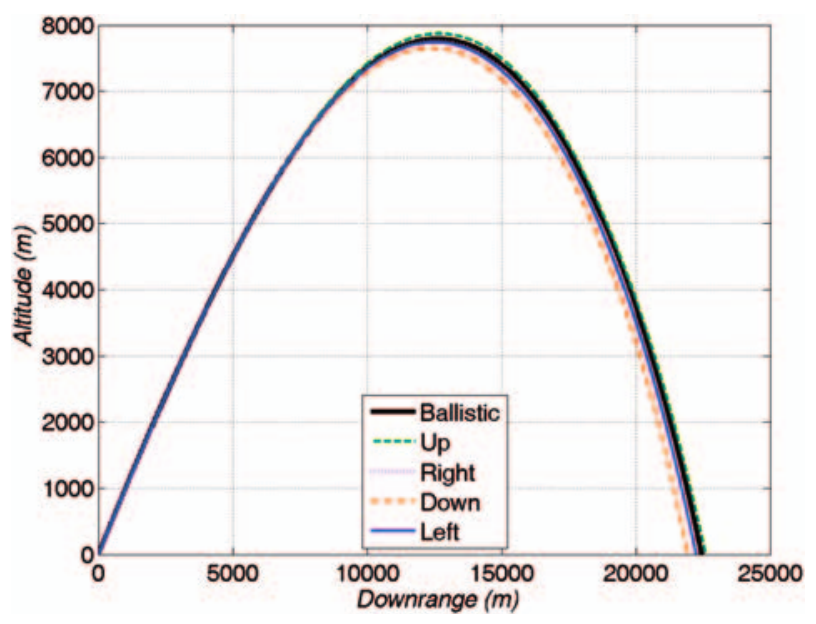

Fig. 8 Altitude trajectory cases fell shorter than the ballistic flight due to the lift-to-drag ratio of the control mechanism. An optimal time for performing the manoeuvre depends on the initial conditions and complex non-linear flight mechanics and aerodynamics of a given flight vehicle. The optimal timing was not investigated in this study; therefore, the control authority was not optimized.

Figure 9 shows the cross-range trajectories with sign reversed to be consistent with Fig. 8. This plot is presented from a birds-eye view; negative crossrange values represent the trajectory bending to the right when viewed from behind the gun. Notice the almost $800 \mathrm{~m}$ of cross-range drift in the ballistic trajectory due to the yaw of repose discussed previously. Comparing the right and left cases shows over $1200 \mathrm{~m}$ in control authority in the cross-range direction. There is more control authority in the cross-range direction than the downrange direction because the up manoeuvre must act against the force of gravity. The up and down cases in Fig. 9 differ slightly from the ballistic flight due to complexities in the angular motion history.

The pitch and yaw angle-of-attack histories are provided in Figs 10 and 11, respectively. All cases have the same pitch and yaw angles-of-attack until the manoeuvre begins at $10 \mathrm{~s}$. In the pitch plane of Fig. 10, the up manoeuvre immediately kicks the angle up to half a degree at $10 \mathrm{~s}$ and the pitch damping then damps the oscillatory pitching motion. These oscillations occur at the pitching rate of the airframe. Oscillatory pitching motion develops again at transonic Mach numbers and grows to about half a degree at subsonic Mach numbers. This coning motion is superposed on top of the approximately half degree pitch angle-of-attack due to the control mechanism. The control-induced angle-of-attack causes the body normal force to

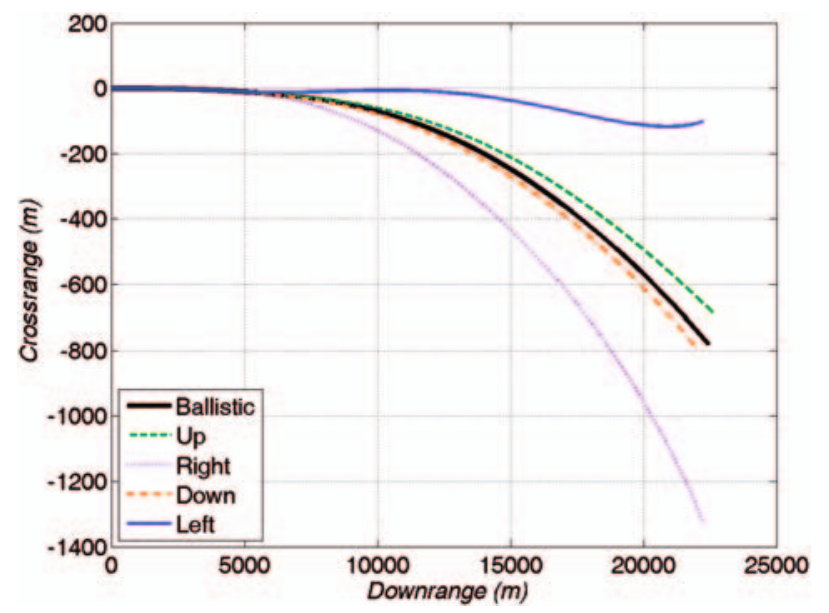

Fig. 9 Cross-range trajectory 
steer the centre of gravity motion of the projectile in the desired roll orientation to remove miss distance.

The pitch angle-of-attack for the down manoeuvre closely mirrors the up case. The significant difference in the pitching motion between the down and up cases is due to yaw of repose. Yaw of repose induces angular motion to pitch the nose up and to the right when viewed from the base of the projectile. Thus, manoeuvres up or right are additive with yaw of repose and manoeuvres down or to the left fight against the yaw of repose and reduce the magnitude of angle-of-attack. Magnus moment is strongly nonlinear with angle-of-attack and when angle-of-attack is lower in general (as seen in comparing the up and down pitch angle-of-attack histories), the Magnusgenerated coning motion is decreased. This is evident in approximately $1^{\circ}$ of coning motion for the up case and half a degree of coning motion for the down case.

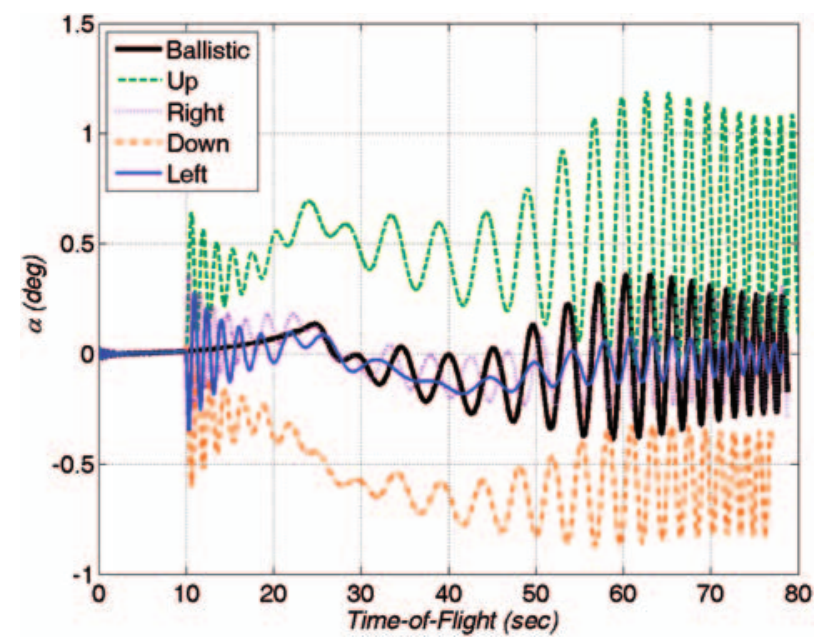

Fig. 10 Pitch angle-of-attack history

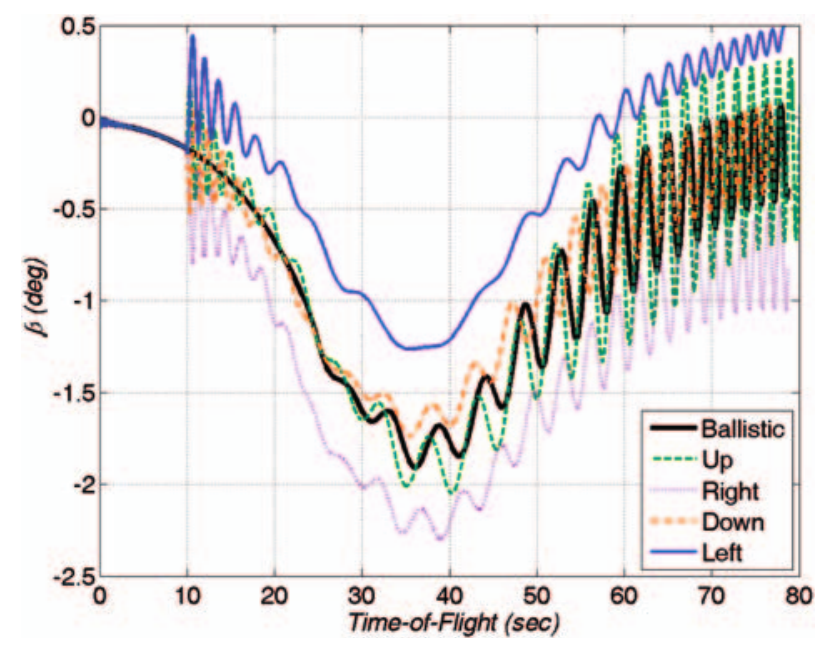

Fig. 11 Yaw angle-of-attack history
Inspecting the yaw angle-of-attack histories in Fig. 11 shows similar phenomena as described for the pitch-of-attack angle. The control mechanism produces angle-of-attack beginning at $10 \mathrm{~s}$ and the action of pitch damping, Magnus moment, and yaw of repose is evident. The interplay of yaw of repose and Magnus moment with the manoeuvre direction generates the magnitude of the coning motion as seen in contrasting the right and left cases. The right manoeuvre increases the yaw angle-of-attack over the ballistic flight to steer the projectile further to the right when viewed from behind the gun as seen in the cross-range plot.

Yaw angle-of-attack is decreased by the left manoeuvre. The left manoeuvre case in Fig. 11 illustrates that the control-induced yaw angle-of-attack never significantly overcomes the yaw of repose (yaw angle-of-attack is positive for only short portions of the flight). The cross-range trajectory for the left case cannot cross to the left of the line of fire (when viewed from behind the gun) since the control-induced angle-of-attack and yaw of repose are at odds.

The pitch-yaw plane angular motion histories for the ballistic and up manoeuvre are presented in Fig. 12. The up case illustrates a different response due to the flow effector, and larger yaw of repose and Magnus-generated coning motion. Inspecting the up trace in Fig. 12 shortly after the manoeuvre begins (see $\alpha=0.6$ and $\beta=-0.2$ ) uncovers scalloped shapes. The circular shapes represent one complete cycle of pitching and yawing motions. The spin rate is larger than the pitch/yaw rate of the projectile. The control mechanism operates at the spin rate. Thus, the scalloped shapes are control mechanism

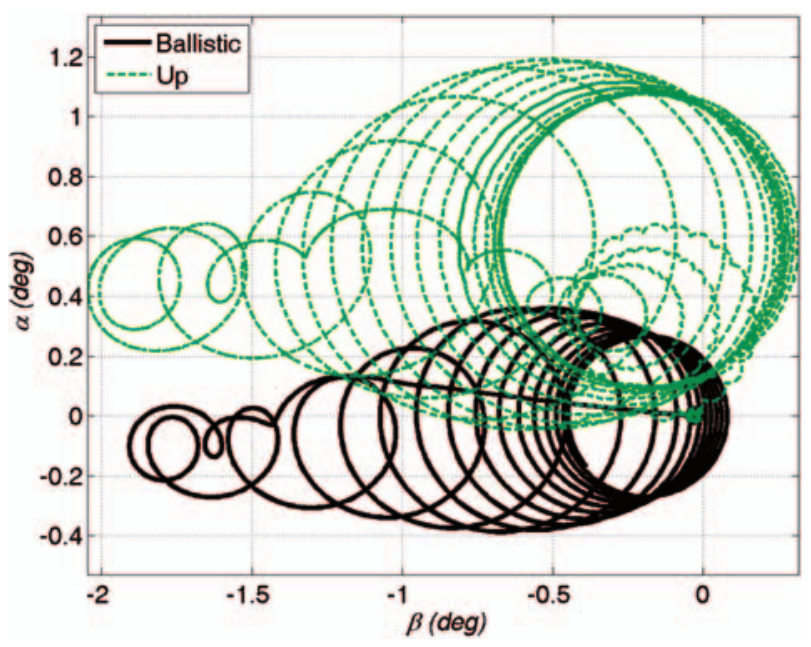

Fig. 12 Angular motion for ballistic and manoeuvring flights 
perturbations to the angular motion within a pitch/ yaw cycle.

The projectile and control mechanism spin rate for the ballistic and up case is provided in Fig. 13. The gun twist and muzzle velocity result in a launch spin rate of $264 \mathrm{~Hz}$. The projectile spin rate decays over the flight for both cases to an impact spin rate near $186 \mathrm{~Hz}$. The inertial and friction of the control mechanism is low enough that the projectile is not spun down noticeably from the ballistic flight. No motor current commands are issued and the control mechanism spin rate stays near $0 \mathrm{~Hz}$ for the ballistic flight. The control mechanism spin rate mirrors the projectile spin rate due to the flight controller. A small transient is evident at $10 \mathrm{~s}$ when the flow effector begins to revolve in and out of the projectile body.

The roll angle of the projectile and control mechanism for the ballistic and up manoeuvre in Fig. 14 is similar to the spin rate history. The only difference between the projectile roll angle for the ballistic and up flight is due to slightly different projectile spin rates. The ballistic control mechanism roll angle is nearly zero. Again, the flight controller tracks the projectile roll angle to produce an equal and opposite control mechanism roll angle. The only difference between the equal and opposite projectile and control mechanism roll angles is the phase angle necessary to steer the projectile in the prescribed roll orientation.

Figure 15 shows the current required to drive the motor for the up manoeuvre (all manoeuvre cases are similar). The current history is similar to the spin rate history; the friction drives the current rather than the coupled dynamics (projectile angular velocity) or motor inertia. Development efforts should focus on reducing the friction in the control mechanism system. The current estimates will be improved as

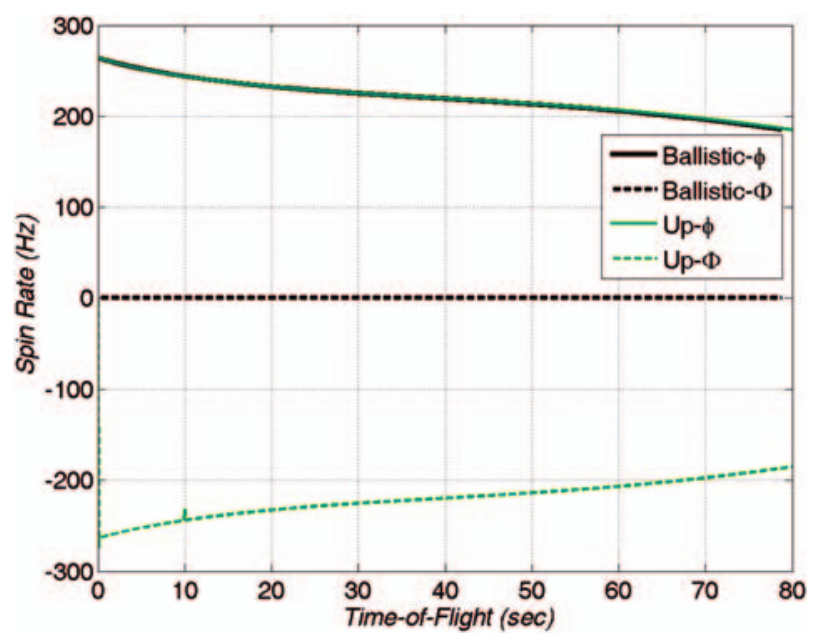

Fig. 13 Body and control mechanism spin rate history more data are available for input to the control mechanism dynamic model. An important aspect to this control mechanism is that the power requirements do not increase with aerodynamic loading. The current does not change at $10 \mathrm{~s}$ when the flow effector first encounters the airstream or vary with the spin cycle. This curve can be integrated to optimize the required gun hardened tactical battery for the smallest possible package. Miniaturization is critical since any projectile volume occupied by GNC components is less available for other subsystems such as warhead.

\section{CONCLUSIONS}

While mature technologies abound for guiding a rocket-propelled missile, currently no customary solution exists for gun-launched precision projectiles. A novel spin-stabilized projectile concept was

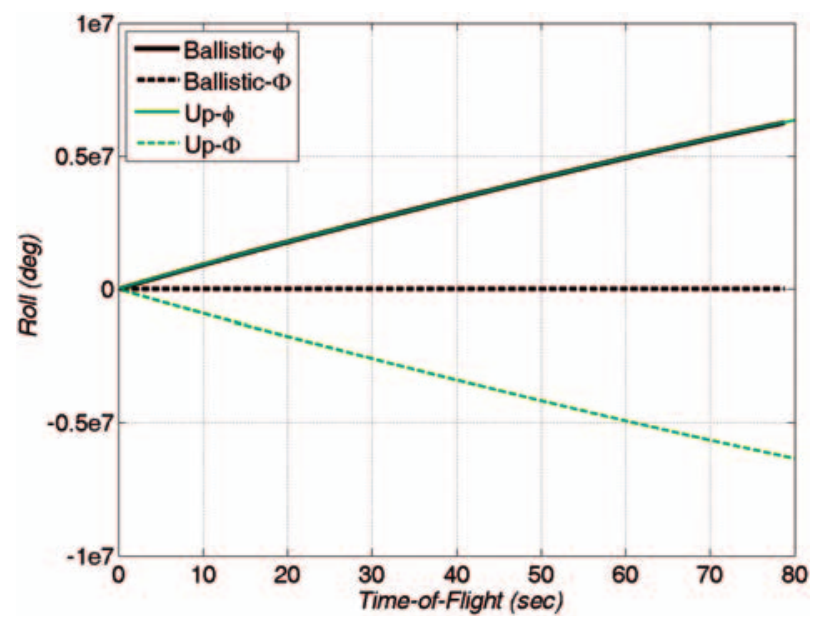

Fig. 14 Body and control mechanism roll angle history

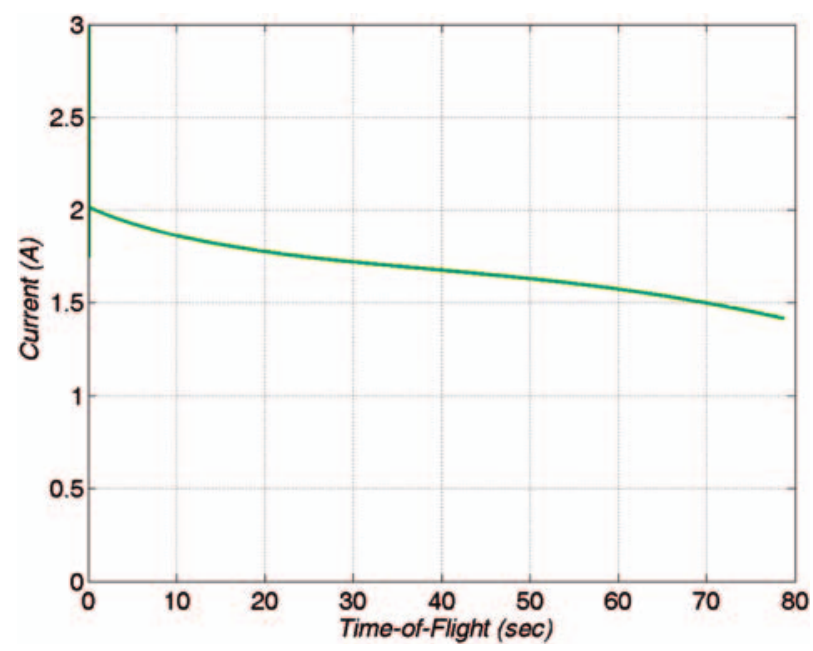

Fig. 15 Estimates of power requirements 
developed in this effort. The non-linear flight mechanics for this multi-body problem were derived from first principles. An aerodynamic model was constructed for this projectile based on experimental and CFD techniques. Verification of the flight dynamics derivation, aerodynamic modelling, and implementation were demonstrated. This airframe was guided through a custom-built flight control law. This analysis enabled the flight stability and response to be examined at the most detailed level.

Results demonstrate the feasibility of this concept for nominal flights of a typical $155 \mathrm{~mm}$ artillery projectile. Control authority estimates are larger than the ballistic dispersion, indicating that guided delivery errors would be driven by the sensors and GNC algorithms and not the inherent airframe manoeuvrability. No dynamic instabilities were encountered. The influence of the flow effector near the boattail of the projectile on the Magnus moment should be explored in greater detail to further substantiate flight stability. Complex angular motion was explained in detail based on the non-linear physics embedded into the flight mechanics and aerodynamic modeling. The effects of the control mechanism, yaw of repose, Magnus moment, pitch damping moment, and projectile drift were elucidated. The flight control law provided satisfactory tracking, as seen in the spin rate and roll orientation results. Power estimates showed that the control mechanism friction drives battery requirements. Future research should focus on experimental investigations on the electromechanical control mechanism system and further aerodynamic characterization and optimization which could supply refined input data to the techniques developed in this effort.

(C) Authors 2011

\section{REFERENCES}

1 Cooper, G. and Costello, M. Flight dynamic response of spinning projectiles to lateral impulsive loads. $J$. Dyn. Syst. Meas. Contr., 2004, 126, 605-613.

2 Davis, B., Malejko, G., Dorhn, R., Owens, S., Harkins, T., and Bischer, G. Addressing the challenges of a thruster-based precision guided mortar munition with the use of embedded telemetry instrumentation. ITEA J., 2009, 30, 117-125.

3 McMichael, J., Lovas, A., Plostins, P., Sahu, J., Brown, G., and Glezer, A. Microadaptive flow control applied to a spinning projectile. In 2nd AIAA Flow Control Conference, Portland, Oregon, 28 June-1 July 2004, AIAA paper no. 2004-2512.

4 Massey, K. and Silton, S. Combining experimental data, computational fluid dynamics, and six-degree of freedom simulation to develop a guidance actuator for a supersonic projectile. J. Aerosp. Eng., 2009, 223, 341-355.

5 Patel, M., Sowle, Z., Ng, T., and Toledo, W. Range and endgame performance assessment of a smart projectile using hingeless flight control. Orbital Research Inc., Cleveland, Ohio, January 2006, AIAA paper no. 2006-671.

6 Costello, M. and Peterson, A. Linear theory of a dual-spin projectile in atmospheric flight. J. Guid. Contr. Dyn., 2000, 23(5), 789-797.

7 Fresconi, F. and Plostins, P. Control mechanism strategies for spin-stabilized projectiles. J. Aerosp. Eng., 2010, 224(G9), 979-991.

8 Frost, G. and Costello, M. Linear theory of a rotating internal part projectile configuration in atmospheric flight. J. Guid. Contr. Dyn., 2004, 27(5), 898-906.

9 Frost, G. and Costello, M. Control authority of a projectile equipped with an internal unbalanced part. J. Dyn. Syst. Meas. Contr., 2006, 128(4), 1005-1012.

10 Rogers, J. and Costello, M. Control authority of a projectile equipped with a controllable internal translating mass. J. Guid. Contr. Dyn., 2008, 31(5), 1323-1333.

11 Soper, W. Projectile instability produced by internal friction. AIAA J., 1978, 16(1), 8-11.

12 Murphy, C. Symmetric missile dynamic instabilities. J. Guid. Contr. Dyn., 1981, 4(5), 464-471.

13 D'Amico, W. Comparison of theory and experiment for moments induced by loose internal parts. J. Guid. Contr. Dyn., 1987, 10(1), 14-19.

14 Hodapp, A. Passive means for stabilizing projectiles with partially restrained internal members. J. Guid. Contr. Dyn., 1989, 12(2), 135-139.

15 Maple, C. G. and Synge, J. L. Aerodynamic symmetry of projectiles. Quart. Appl. Math., 1949, VI(4), 345-366.

16 Whyte, R., Hathaway, W., and Friedman, E. Analysis of free flight transonic range data of the $155 \mathrm{~mm}$, M483A1, and XM795 projectiles. U.S. Army Research Lab., ARLCD-CR-79016, Aberdeen Proving Ground, Maryland, August 1979.

\section{APPENDIX}

\section{Notation}

$\vec{a}_{C / I}$

acceleration of the control

mechanism centre of gravity with respect to an observer in the inertial frame

$\vec{a}_{P / I} \quad$ acceleration of the projectile centre of gravity with respect to an observer in the inertial frame

$C_{l_{p}} \quad$ roll damping coefficient

$C_{m_{\alpha}} \quad$ pitching moment derivative coefficient

$C_{m_{q}}+C_{m_{\dot{\alpha}}}$ $C_{m C}$ pitch damping coefficient pitching moment coefficient for control 


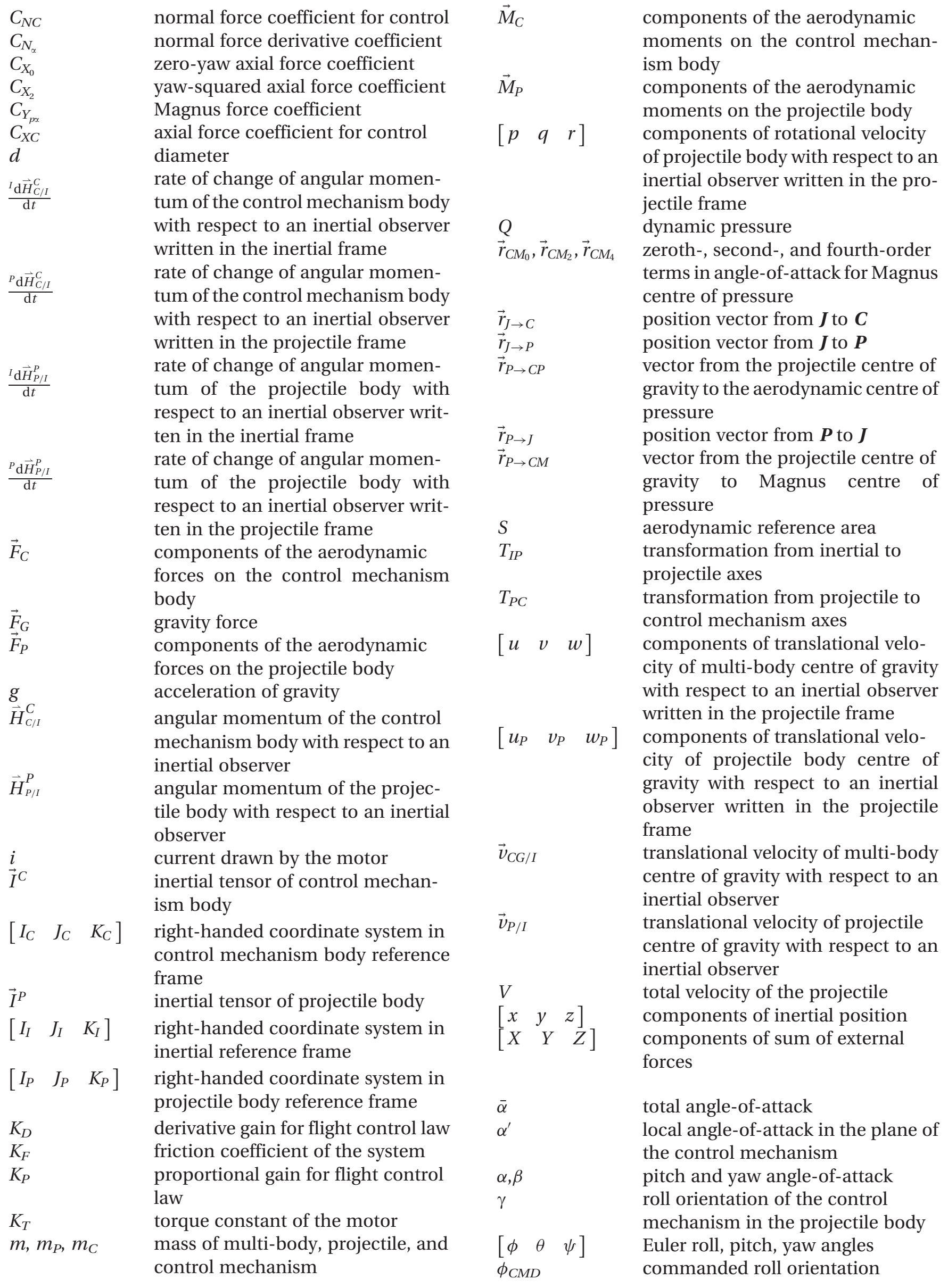




$\begin{array}{llll}\Phi & \begin{array}{l}\text { roll angle of control mechanism } \\ \text { rotational velocity of control } \\ \text { mechanism body with respect to an }\end{array} & \Omega & \begin{array}{l}\text { rotational rate of control } \\ \text { mechanism }\end{array} \\ & \begin{array}{l}\text { inertial observer } \\ \text { rotational velocity of projectile } \\ \text { body with respect to an inertial } \\ \text { observer }\end{array}\end{array}$

